\title{
Topic issue on new treatments in bladder cancer
}

\author{
J. A. Witjes
}

Received: 30 January 2009 / Accepted: 2 February 2009 / Published online: 20 February 2009

(c) The Author(s) 2009. This article is published with open access at Springerlink.com

This topic issue of the world journal of urology deals with new treatments in non muscle invasive bladder cancer (NMIBC). NMIBC is a frequent, important and costly disease. After several decades in which no or only few new developments were noted, NMIBC is currently a disease where several studies with new drugs and drug applications are tested.

In the first manuscript, Ploeg and coworkers look at the present and future burden of urinary bladder cancer (UBC) worldwide. UBC occurrence is about three to four times higher in developed countries. This is caused by differences in exposure to carcinogens like smoking, but also by differences in registration. For example, NMIBC is not always included in the registration. Still, in both the USA and Western Europe, the lifetime probability to develop UBC (pTa included) is more than 4 and $1.2 \%$ in males and females, respectively. This obviously translates in a high number of patients alive with bladder cancer at a specific point in time. With the "duration" of the disease estimated to be 7.5 years, and 357,000 new cases worldwide each year, the prevalence can be estimated at almost 2.7 million. In developed countries the incidence is decreasing, predominantly due to decreasing smoking habits and better industrial circumstances, in spite of aging of the population. In the developing countries incidence will increase due to more smoking and industrialization. In all, bladder cancer is a significant and increasing health problem.

Obviously, this increasing problem is a costly problem. Sievert and coworkers looked at the economic side of bladder

J. A. Witjes $(\square)$

Department of Urology (659),

Radboud University Nijmegen Medical Centre, PO Box 9101, 6500 HB Nijmegen, The Netherlands e-mail: f.witjes@uro.umcn.nl cancer diagnosis and treatment. UBC is one of the most costly cancers, and has the highest lifetime treatment costs per patient of all cancers. The high recurrence rate of NMIBC and the subsequent need for follow up are important issue in the economic and human burden of this disease. New possibilities to reduce costs are urinary markers, outpatient transurethral resection (TUR), better intravesical therapy and the use of photodynamic diagnosis (PDD). Many new developments initially induce more costs. Urinary markers, although improving sensitivity, still cannot replace cystoscopy. PDD, clearly improves diagnosis and treatment as described elsewhere in this topic issue, and also lowers long term costs as shown with models in several countries. Finally, the training of urologic surgeons must be improved as initial therapy has a profound impact on the patient and total $\mathrm{BC}$ costs. A renewed interest and investment in $\mathrm{BC}$ research would be essential to ensure future advancements.

In the third manuscript, Goh and Lerner discuss current and new technology in bladder cancer diagnosis. The current standard, white light cystoscopy has significant limitations. Even under optimal conditions many lesions are overlooked. In high risk tumors re-resection reveals 40 $60 \%$ residual tumor, and in case of CIS also up to $50 \%$ is missed. A significant improvement has been achieved with fluorescence cystoscopy. Both 5-ALA and hexyl 5-ALA (HAL) have been studied extensively, and improve diagnostic accuracy with approximately $20-25 \%$. This results in a more complete TUR, better treatment and lower recurrence rates. Limitations are amongst others a small increase in the false positive rate, and photobleeching, something that is not a problem with Hypericin. Hypericin, however, has low solubility, and is still under investigation. Optical coherence tomography (OCT) has a high resolution and it permits micro-architectural detection to a depth of 1-2 mm. 
This might enhance diagnosis and staging, but this also has to be studied in larger studies. Narrow-band imaging (NBI) is another new imaging technique, which utilizes a restricted optical spectrum which better detects (small) blood vessels. An obvious limitation is that hyper-vascularity is not cancer specific. Virtual cystoscopy, finally, uses reconstructed imaging data to display the urinary tract. An advantage is that it is not invasive, disadvantages are the limited detection of smaller lesions and CIS, there is no morphologic information and it does not allow simultaneous intervention. In all, newer techniques will certainly improve the (endoscopic) diagnosis of bladder cancer in the coming years.

Not only seeing a bladder tumor is of importance, but also the initial operation should be optimal. Unfortunately, a TUR is not always appreciated as a real operation, and textbook information on TUR techniques is sparse. Wilby and coworkers have looked at the technique of TUR and also at new TUR techniques. Current golden standard TUR techniques, first described in 1931, are associated with unacceptably high early recurrence rates, probably due to incomplete resection in a substantial number of these cases. Early second resection has shown to find residual tumors in $25-40 \%$ and has been shown to reduce recurrence rates, and increase response to intravesical therapy. It should be considered in high risk NMIBC in case of bladder conservation. Some techniques from TURP might also be used in the bladder. Bipolar resection gives less tissue damage and less obturator nerve reaction. Laser resection could facilitate day case management in some patients. As mentioned above, fluorescence cystoscopy also significantly improves the quality of TUR. Finally, the traditional 'incise and scatter' resection technique goes against all oncological surgical principles. En-bloc resection of tumors could be far better and is currently subject of further development and evaluation. In all, the golden standard TUR needs to evolve since residual tumor and recurrence rates of post TUR are unacceptably high.

However, even with better diagnosis and TUR technique adjuvant therapy remains necessary. Currently used drugs, such as MMC and BCG, are on the market for several decades. New drugs are needed and studied, but there is an increasing need to study efficacy before testing new drugs in humans. Arentsen and coworkers give an overview of preclinical testing of new intravesical therapeutic agents. The ideal bladder tumor model should grow intravesically in an immunocompetent and large enough animal and should be a urothelial cell carcinoma (UCC) in different stages. Finally it should be easy to develop and to reproduce. Rat models, therefore, are preferred over murine models, as are intravesical models over subcutaneous models. Indeed intravesical rat UCC models exist, which can even be monitored with cystoscopy. Remaining challenges are tumor cell implantation methods, follow up monitoring and the short period the tumors are non invasive and suitable for intravesical treatment testing.

One of the new treatments that have been tested in animals is intravesical apaziquone. Hendricksen and coworkers report their 2-year follow-up of a phase II marker lesion study with apaziquone. In 46 patients, all but one non muscle invasive tumors were resected, and apaziquone was given weekly for 6 weeks. Short term side effects were mild, long term side effects absent. The initial 3 months histologically confirmed complete response rate was $31 / 46$ $(67.4 \%)$. The intention-to-treat analysis showed that $49.5 \%$ of the complete responders remained free of recurrence at 24 months follow up. The overall recurrence-free survival was $39 \%(18 / 46)$ at 24 months follow up. In conclusion, the initial high complete response rate and subsequent recurrence free survival in patients with this risk profile indicates that apaziquone is a potent drug, and further studies are ongoing.

Not only new drugs are under investigation, but also new treatment modalities are being studied. Di Stasi and Riedl report on their experience with electromotive drug administration (EMDA). The principles are described. The concept is that EMDA increases local efficacy of drugs by enhancing the transmembranous transport into tissue. The combination of EMDA and MMC in vitro indeed showed a fourto sevenfold increase in bladder wall MMC concentration over passive diffusion (PD). In human studies, these higher MMC concentrations were shown to result in lower recurrence rates than $\mathrm{PD}$ in randomized studies. A subsequent study in CIS patients showed that EMDA/MMC was at least as effective as BCG. Both treatments were superior to PD with MMC with regard to response and recurrence rates, not with regard to progression. Toxicity of EMDA/ MMC was between that of BCG and PD/MMC. Although combinations of BCG and MMC have been studied, results are inconclusive. However, in a randomized study with 212 patients and 88 months median follow up, the combination of BCG and EMDA/MMC showed superior outcome compared to BCG alone for all endpoints, including progression and disease specific survival.

A new concept is pre-TUR EMDA/MMC. In a randomized trial with 167 patients, pre-TUR EMDA/MMC was significantly better than TUR follow by one immediate instillation with MMC or TUR alone. In all, in spite of modestly increased costs, the use of EMDA appears a significant improvement in these initial studies in the treatment of several risk categories of patients with NMIBC, and should be studied further.

Difficult patients to treat are those refractory to BCG. Guidelines advise cystectomy for these patients, but not all are willing of fit enough to undergo major surgery. Therefore, especially in these patients, new approaches are needed. 
Barlow and coworkers have updated their experience with intravesical docetaxel in BCG refractory patients. They report on 33 patients treated in and after a phase I trial. Side effects appear to be extremely mild. A total of 20 patients achieved a complete response, 10 of which received maintenance docetaxel. The 1- and 2-year recurrence free rates were 45 and $32 \%$ respectively, with a tendency to better results in the highest used dose $(75 \mathrm{mg} /$ $100 \mathrm{~mL}$ ) and maintenance treatment. This makes docetaxel a promising and safe alternative in these patients, worth further study.

Witjes and coworkers report on intravesical thermo-chemotherapy (TCT) in (predominantly) BCG refractory CIS patients. TCT has proven its value as adjuvant therapy in intermediate and high risk patients. Also as second line treatment TCT has shown promising effects. In this retrospective analysis, 51 patients with CIS are reported of which 34 were treated before with BCG. TCT was done with $\mathrm{MMC}$ and a temperature range between 41 and $44^{\circ} \mathrm{C}$. The schedule was a six weekly induction course followed by maintenance therapy up to 1 year. Side effects were mild and predominantly transient bladder complaints. A total of 49 patients were evaluable, and 45 had a biopsy and cytology proven complete response (92\%). In two additional patients CIS disappeared, but they had persistent papillary lesions. After a mean follow up of 27 months, 22 of the 45 complete responders (49\%) experienced recurrent tumor, of which 4 had tumor progression to invasive bladder cancer. In all, TCT seems an effective and safe treatment, even in patients with BCG refractory CIS.

A third option for patients failing BCG is the combination of BCG and interferon alpha (IFNA), as described by Nepple and coworkers. In vitro studies have shown synergism between IFNA and BCG. In their analysis the 2 year disease free rate in 467 BCG failing patients was $45 \%$, as compared to $59 \%$ in BCG naive patients. Recent findings, however, suggest that in BCG naive patients the additional effect of IFNA is at the best limited. In BCG failures, IFNA combined with BCG may salvage especially single-course BCG failures or late BCG relapsers, while patients relapsing quickly may be destined to also fail this combination.

Finally, Hautmann and coworkers address the role of cystectomy in T1G3 patients, which indeed is in the EAU guidelines as potential therapy in these patients. One of the comparisons they make is the long term outcome in patients with an initial T1 G3 tumor $(n=124)$ treated with radical cystectomy within 90 days after TUR in their institution, versus 99 patients that had T1G3 recurrence after initial conservative therapy, that were subsequently referred to Ulm for radical cystectomy. Upstaging from the TUR to the cystectomy specimen was found in $29.1 \%$ in the patients with an immediate cystectomy, versus $63.6 \%$ for those with a cystectomy at recurrence. Also the percentage of non organ confined disease and positive nodal disease was more frequent in the deferred cystectomy patients. Most importantly, tumor specific survival after 10 years was 78.9 versus $64.5 \%$ in favor of early cystectomy. In spite of the obvious limitations of a retrospective study, as nicely pointed out by the authors, a randomized trial is not available. This means that results from expert centers like Ulm might very well be the best we will ever have. In all, these results seem to support the EAU guideline advice that a cystectomy is one of the treatment options in T1G3 patients.

In summary, the above manuscripts give an insight in current limitations and subsequent developments in the diagnosis and treatment of NMIBC. Moreover, the practicing urologist will definitely find practical advises for high risk and BCG failing patients.

Open Access This article is distributed under the terms of the Creative Commons Attribution Noncommercial License which permits any noncommercial use, distribution, and reproduction in any medium, provided the original author(s) and source are credited. 